\title{
Scheinselbstständigkeit in der osteopathischen Praxis
}

Osteopathen werden häufig in osteopathischen Praxen von dem Praxisinhaber als freie Mitarbeiter beschäftigt oder auf anderer rechtlicher Basis als selbstständig eingestuft. In der Konsequenz zahlt der Praxisinhaber keine Sozialversicherungsabgaben und der Mitarbeiter sorgt (im Idealfall) selbst für seine Absicherung im Kranken- und Rentenfall. Wird jedoch das Beschäftigungsverhältnis bei einer Überprüfung entgegen der Einstufung durch die Vertragsparteien als abhängiges Beschäftigungsverhältnis eingestuft, drohen dem Praxisinhaber wegen der unterlassenen Zahlung an Sozialversicherungsbeiträgen hohe Nachforderungen und weitere erhebliche rechtliche Nachteile.

In dem folgenden Beitrag werden zunächst die Begrifflichkeiten im Einzelnen erklärt und definiert, was Scheinselbstständigkeit bedeutet. Des Weiteren werden die Indizien aufgeführt, die zur Prüfung, ob eine Scheinselbstständigkeit vorliegt, herangezogen werden. Die sich anschließenden Beispielfälle der Rechtsprechung und Konsequenzen der Scheinselbstständigkeit sollen die praktischen Konsequenzen verdeutlichen. Neben der Möglichkeit einer Statusfeststellung werden zum Abschluss die verschiedentlich diskutierten Lösungsmöglichkeiten erörtert.

\section{Verschiedene Formen der Tätigkeit}

\section{Abhängige Beschäftigung}

Nach § 7 Abs. 1 SGB IV ist die (abhängige) Beschäftigung die nicht selbstständige Tätigkeit, insbesondere in einem Arbeitsverhältnis. Eine abhängige Beschäftigung wird daher angenommen, wenn eine Person aufgrund eines privatrechtlichen Vertrags für andere unselbstständige Dienste erbringt. Kennzeichen der abhängigen Beschäftigung ist die persönliche Abhängigkeit des Angestellten von dem Arbeitgeber. Nach § 7 Abs. 1 Satz 2 SGB IV liegt dies beispielsweise vor, wenn der Beschäftigte in den Praxisbetrieb eingegliedert ist und dabei einem Weisungsrecht des Ar- beitgebers unterliegt, das Zeit, Dauer und Ort der Ausführung umfasst [1].

\section{Selbstständigkeit}

Die in vielen Praxen beschäftigten freien Mitarbeiter gehen meist davon aus, selbstständig tätig zu sein. Sozialversicherungsabgaben werden daher nicht durch den Praxisinhaber geleistet. Nach der höchstrichterlichen Rechtsprechung ist die Selbstständigkeit vorrangig gekennzeichnet durch das eigene Unternehmerrisiko des selbstständig Tätigen. Zudem ist sie üblicherweise durch folgende Merkmale charakterisiert: das Vorhandensein einer eigenen Betriebsstätte und die Verfügungsmöglichkeit über die eigene Arbeitskraft und die im Wesentlichen frei gestaltete Tätigkeit und Arbeitszeit [2].

\section{Scheinselbstständigkeit}

Scheinselbstständig ist ein Arbeitnehmer, der nur zum Schein als Selbstständiger auftritt, nach den Gesamtumständen aber als abhängig beschäftigt einzustufen ist. Bei der Frage, ob jemand Scheinselbstständiger ist oder nicht, geht es daher im Kern um die Abgrenzung zwischen der abhängigen Beschäftigung einerseits und der Selbstständigkeit andererseits.

Ausgangspunkt der Prüfung der Scheinselbstständigkeit ist zunächst das Vertragsverhältnis der Beteiligten, wenn ein solches schriftlich niedergelegt ist. Ist der freie Mitarbeiter also nach dem schriftlichen Vertrag tatsächlich weisungsfrei, nicht in den Praxisbetrieb eingegliedert und verfügt über ein unternehmerisches Risiko? Spricht der Vertrag für eine Selbstständigkeit, wird weiter geprüft, ob die tatsächliche Umset- 
zung des Vertrags im Widerspruch zu der getroffenen Vereinbarung steht, ob es sich also tatsächlich nur um einen „Etikettenschwindel" handelt.

\section{DEFINITION}

Das vertraglich Vereinbarte bzw. der Wortlaut des Vertrags tritt hinter den tatsächlichen Umständen zurück, wenn der Vertrag nicht der gelebten Praxis entspricht [3]. Die Einstufung des freien Mitarbeiters als abhängig beschäftigt oder selbstständig erfolgt grundsätzlich nicht nach dem Willen der Vertragspartner, sondern wird objektiv nach den Gesamtumständen des Einzelfalls festgestellt [4].

Hierbei sollen nach der Vorgabe der höchstrichterlichen Rechtsprechung alle in dem Einzelfall als Indizien in Betracht kommenden Umstände festgestellt, in ihrer Tragweite zutreffend erkannt, gewichtet und abgewogen werden [5]. Keiner der Umstände für sich ist als K-.o.-Kriterium für die Entscheidung der prüfenden Behörde bzw. des prüfenden Gerichts alleine ausschlaggebend. Häufig sprechen einige Kriterien für eine abhängige Beschäftigung, andere für eine selbstständige Tätigkeit. Ob jemand abhängig beschäftigt oder selbstständig tätig ist, hängt dann maßgeblich davon ab, welche der Indizien in dem relevanten Einzelfall überwiegen [6].

\section{Abgrenzung: Selbst- ständigkeit und abhängige Beschäftigung}

Im Folgenden werden die Indizien dargestellt, die in einer osteopathischen Praxis jeweils für eine abhängige Beschäftigung des freien Mitarbeiters, somit also für seine Scheinselbständigkeit sprechen, sowie auch die Indizien angegeben, die seine Selbstständigkeit angeben.

\section{Indizien für die abhängige Beschäftigung}

Für das Weisungsrecht des Praxisinhabers und damit für die Scheinselbstständigkeit des freien Mitarbeiters sprechen folgende Indizien:
- Dem freien Mitarbeiter ist es untersagt, seine Arbeitsleistung auf andere Personen zu übertragen.

- Der freie Mitarbeiter ist nur für einen Auftraggeber tätig.

- Der Erstkontakt der Patienten findet ausschließlich über den Praxisinhaber statt.

- Der Praxisinhaber vermittelt Patienten an den freien Mitarbeiter, der Mitarbeiter kann die Patienten nicht ablehnen.

- Das Praxisinventar wird durch den Praxisinhaber gestellt.

- Nur der Praxisinhaber tritt nach außen als Praxisbetreiber auf. Der freie Mitarbeiter nutzt den Webauftritt des Praxisinhabers und wird auf der Webseite des Praxisinhabers als Teammitglied mit aufgeführt.

- Der freie Mitarbeiter hat keine eigenen Werbemittel, firmiert mit dem Logo des Praxisinhabers.

- Der freie Mitarbeiter schließt die Behandlungsverträge mit Patienten im Namen des Praxisinhabers ab, die Rechnungsstellung erfolgt durch die Praxis an Patienten, die Bezahlung erfolgt auf das Konto des Praxisinhabers.

- Der freie Mitarbeiter unterhält keine eigene Praxiskartei.

- Die Archivierung abgeschlossener Behandlungskontakte erfolgt nur über den Praxisinhaber [7].

- Der freie Mitarbeiter hat keine eigene Praxis/Betriebsstätte.

- Arbeitsmittel wie Liegen, Handtücher, Bestuhlung für die Wartezeit und Ähnliches werden von dem Praxisinhaber gestellt.

- Der freie Mitarbeiter hat keine eigene Haftpflichtversicherung.

- Der freie Mitarbeiter beschäftigt keine eigenen Arbeitnehmer.

- Der freie Mitarbeiter bezieht eine im Wesentlichen vom Gewinn und Verlust unabhängige Vergütung.

- Der freie Mitarbeiter war in der Vergangenheit für die gleiche Tätigkeit als $\mathrm{Ar}$ beitnehmer bei dem Praxisinhaber beschäftigt.

Für die Eingliederung in den Praxisablauf und somit für die abhängige Beschäftigung sprechen folgende Gesichtspunkte:

- Der freie Mitarbeiter ist in die Praxisorganisation eingegliedert.
- Der Auftraggeber trägt als Praxisinhaber die fachliche Verantwortung für die Behandlung der Patienten.

- Der freie Mitarbeiter muss sich einem von dem Praxisinhaber vorgegebenen Vertragswerk ohne eigenen Gestaltungsspielraum unterwerfen.

- Der Praxisinhaber regelt den Arbeitsablauf auch für den freien Mitarbeiter.

- Der Praxisinhaber gibt die Zeiteinteilung des freien Mitarbeiters vor.

- Der Kontakt der Patienten zu dem freien Mitarbeiter erfolgt über die Praxis.

- Der Praxisinhaber gibt die Preisgestaltung vor.

\section{Indizien für die Selbstständigkeit}

Folgende Indizien sprechen für die Weisungsfreiheit des freien Mitarbeiters und damit für seine Selbstständigkeit:

- Der freie Mitarbeiter hat Entscheidungsfreiheit über Ort und Zeit der Leistung.

- Er hat auch Entscheidungsfreiheit über die Preisgestaltung.

- Der freie Mitarbeiter sorgt selbst für die Terminvereinbarungen, -verlegungen und -absagen mit den Patienten.

- Der freie Mitarbeiter ist im Einsatz von Hilfskräften frei und kann weitere Mitarbeiter beschäftigen.

- Der freie Mitarbeiter arbeitet für mehr als nur 1 Auftraggeber.

- Der freie Mitarbeiter verfügt über eigene Patientendateien, die von der übrigen Praxis getrennt sind.

- Der freie Mitarbeiter sorgt selbst für die administrativen Tätigkeiten, z. B. für die Rechnungsstellung und das Mahnwesen.

- Der freie Mitarbeiter verfügt über eine eigene Praxisausstattung.

- Der freie Mitarbeiter nutzt für die Durchführung seiner Tätigkeit, (insbesondere) soweit er Hausbesuche tätigt, einen eigenen Pkw und sorgt für die Kosten des Betriebs des Pkw selbst.

Für ein Unternehmerrisiko und damit für die Selbstständigkeit des freien Mitarbeiters sprechen folgende Indizien:

\footnotetext{
Merke

Maßgebendes Kriterium für ein unternehmerisches Risiko ist, ob eigenes Kapital oder die eigene Arbeitskraft auch mit der Gefahr des Verlusts eingesetzt
} 
wird und somit der Erfolg des Einsatzes also ungewiss ist [8].

- Der freie Mitarbeiter nutzt eigene finanzielle Mittel in der Praxiswerbung und Außendarstellung und verfügt über eine eigene Außendarstellung/ein eigenes Logo.

- Der freie Mitarbeiter ist am wirtschaftlichen Erfolg der Praxis nicht eigenständig und unabhängig vom Ausmaß des eigenen persönlichen Arbeitseinsatzes beteiligt. Das Risiko des Verlusts der Vergütung bei Ausbleiben eines Patienten oder Nichtzahlung reicht der Rechtsprechung für das Unternehmerrisiko nicht aus! (vgl. BSG, Urteil vom 24.03.2016, Az. B 12 KR 20/14 R)

- Der freie Mitarbeiter nimmt eine eigene Kundenakquise vor.

- Der freie Mitarbeiter tritt selbst im Hinblick auf die geprüfte Tätigkeit unternehmerisch am Markt auf. Eine Bewerbung einer anderen Tätigkeit in anderer Praxis ist hier nicht ausschlaggebend [9].

- Der freie Mitarbeiter schließt die Behandlungsverträge mit den Patienten in eigenem Namen, die Bezahlung erfolgt auf das Konto des freien Mitarbeiters.

- Der freie Mitarbeiter erhält keinen festen Stundensatz bzw. ein monatliches festes Entgelt, sondern eine prozentuale Vergütung abhängig von der Zahl der behandelten Patienten.
- Der freie Mitarbeiter hat zusätzlich noch eine weitere Betriebsstätte/Praxis bzw. eigene Behandlungsräume.

- Der freie Mitarbeiter beschäftigt eigenes Personal, das ihn in Abwesenheitsphasen auch vertritt.

\section{Merke}

Wichtig ist zudem Folgendes: Die Tatsache, ob ein freier Mitarbeiter noch in anderen Praxen beschäftigt ist, ist nach der neueren Rechtsprechung nicht ausschlaggebend.

Beurteilt werden nur die konkreten Umstände der Tätigkeit in der jeweiligen Praxis [10].

\section{Beispielfälle}

In einer Entscheidung aus Niedersachsen forderte die Rentenversicherung im Rahmen einer Betriebsprüfung eine Physiotherapiepraxis auf, Gesamtsozialversicherungsbeiträge in Höhe von 27262,63€ nachzuzahlen (inklusive Säumniszuschläge von 6656,50€), da die Merkmale einer selbstständigen Tätigkeit nicht gegeben waren. Die freie Mitarbeiterin verfügte weder über eigene Betriebsräume noch beschäftigte sie eigene Arbeitnehmer. Die Vergütung erfolgte über die Praxisinhaberin. Das Landessozialgericht hat in 2. Instanz entschieden, dass zwar auch zahlreiche Merkmale einer selbstständigen Tätigkeit vorlägen. Es überwögen aber die ty- pusbildenden Merkmale, die für eine sozialversicherungspflichtige, abhängige Beschäftigung sprechen. Benannt wurden für Letzteres die Ausgestaltung des Vertrags, die Eingliederung in den Praxisbetrieb, die fehlende eigene Betriebsstätte und das fehlende Unternehmerrisiko. Diese Entscheidung wurde durch das Bundessozialgericht bestätigt [11].

Für viel Unruhe hat auch eine Entscheidung des Bayerischen Landessozialgerichts aus dem Jahr 2014 gesorgt: In einer Physiotherapiepraxis in München forderte die Rentenversicherung nach einer Betriebsprüfung von dem Praxisinhaber wegen 2 als fälschlicherweise selbstständig deklarierten freien Mitarbeitern einen Betrag von 46383,50€ an Sozialversicherungsbeiträgen nach. Bei der zu erfolgenden Abwägung fand die Rentenversicherung es zunächst ausschlaggebend, dass die Praxis über den Praxisinhaber kassenzugelassen war, die freien Mitarbeiter jedoch keine eigene Kassenzulassung hatten. Zudem wurden den freien Mitarbeitern die Betriebseinrichtungen zur Verfügung gestellt, Therapieart und -dauer waren von dem Praxissystem vorgegeben, in welches die freien Mitarbeiter eingebunden waren. Die für das Vorliegen einer Selbstständigkeit sprechenden Merkmale wurden als nicht überwiegend angesehen [12].

Auch für einen Zahnarzt hat das Landessozialgericht Niedersachsen-Bremen erst 
kürzlich bestätigt, dass dieser nach der zu erfolgenden Gesamtabwägung abhängig beschäftigt war, da er nicht an dem Gewinn oder Verlust der Praxis, die ihn auf Basis eines Kooperationsvertrags beschäftigte, beteiligt war, damit kein Unternehmerrisiko trug und auch nach den weiteren tatsächlichen Umständen in die Praxisabläufe eingegliedert war [13].

\section{Folgen der Scheinselbst- ständigkeit}

\section{Arbeitsrecht}

Scheinselbstständige gelten rechtlich als Arbeitnehmer. Aus diesem Grunde stehen ihnen auch sämtliche Rechte zu, welche das Arbeitsrecht für Arbeitnehmer vorhält, wobei vorrangig die folgenden zu benennen sind:

- das Recht auf Entgeltfortzahlung im Krankheitsfall,

- das Recht auf bezahlten Erholungsurlaub von mindestens 4 Kalenderwochen pro Kalenderjahr,

- Kündigungsschutz nach dem Kündigungsschutzgesetz sowie weitere Schutzvorschriften zugunsten Schwangerer und Schwerbehinderter.

\section{Sozialversicherungsrecht}

Wird festgestellt, dass zwischen den Parteien ein abhängiges Beschäftigungsverhältnis bestand, hat dies auch sozialversicherungsrechtlich drastische Folgen für den Arbeitgeber:

- Abführen künftiger Sozialversicherungsbeiträge: Wird das Beschäftigungsverhältnis fortgeführt, fallen die regulären Sozialversicherungsbeiträge auf das beitragspflichtige Bruttoarbeitsentgelt an. Alleiniger Schuldner für das Abführen der Sozialversicherungsbeiträge gegenüber dem Sozialversicherungsträgers ist der Arbeitgeber. Nur im Innenverhältnis hat der Arbeitnehmer die Hälfte zu tragen, im Außenverhältnis haftet der Arbeitgeber jedoch allein.

- Nachentrichten bisheriger Gesamtsozialversicherungsbeiträge: Der Arbeitgeber schuldet für den zurückliegenden Zeitraum die angefallenen Gesamtsozialversicherungsbeträge als alleiniger Schuldner. Diese Nachforderung bezieht sich hierbei sowohl auf den Arbeitgeber- als auch den Arbeitnehmeranteil. Die Verpflichtung zum Nachzahlen der Gesamtsozialversicherungsbeiträge verjährt 4 Jahre nach $A b-$ lauf des Kalenderjahrs, in dem sie fällig geworden sind (§ 25 Abs. 1 Satz 1 SGB IV). Hat der Arbeitgeber vorsätzlich gehandelt, gilt eine Verjährungsfrist von 30 Jahren. Es reicht hierbei ein sogenannter bedingter Vorsatz, d.h., es reicht aus, wenn der Arbeitgeber die Pflicht zur Zahlung von Sozialversicherungsleistungen für möglich gehalten hat und den Rechtsverstoß „billigend in Kauf genommen hat".

- Regressansprüche gegenüber dem freien Mitarbeiter? Im Innenverhältnis ist ein Anspruch gegen den Arbeitnehmer/freien Mitarbeiter nur in Höhe des Arbeitnehmeranteils möglich. Der Erstattungsanspruch ist aber erheblich beschränkt: Denn der Anspruch kann nur durch Abzug vom Arbeitsentgelt geltend gemacht werden, indem der Arbeitgeber mit seinem Erstattungsanspruch gegen die Lohnforderung des Arbeitnehmers aufrechnet und dies im Regelfall nur bei den 3 nächsten Lohnund Gehaltszahlungen.

\section{Merke}

Regressmöglichkeiten gegen freie Mitarbeiter bestehen so gut wie keine.

\section{Statusfeststellungsverfahren}

Zur Klärung des Status' des freien Mitarbeiters wird häufig erwogen, ein sog. Statusfeststellungsverfahren durchzuführen, um hiermit Rechtssicherheit zu erlangen.

\section{Arbeitsrecht}

Im Arbeitsrecht kann das Vorliegen bzw. Nichtvorliegen eines Arbeitsverhältnisses durch die Klage auf Feststellung des Bestehens eines Arbeitsverhältnisses beim zuständigen Arbeitsgericht geklärt werden.

\section{Sozialversicherungsrecht}

Um den Status des freien Mitarbeiters im Sozialversicherungsrecht klären zu lassen, können die Vertragspartner nach §7a Abs. 1 Satz 1 SGB IV die sogenannte Clearingstelle der Rentenversicherung anrufen und eine Entscheidung über den Status der beschäftigten Person beantragen. Die- ser Antrag kann nur dann gestellt werden, wenn die Rentenversicherung nicht bereits ein Verfahren zur Feststellung einer abhängigen Beschäftigung eingeleitet hat.

Wichtig ist hierbei: Wird der Antrag innerhalb eines Monats nach Aufnahme der Tätigkeit gestellt und hat der freie Mitarbeiter für den Zeitraum zwischen Aufnahme der Beschäftigung und der Entscheidung eine Absicherung gegen das finanzielle Risiko von Krankheit und zur Altersvorsorge vorgenommen, welche der Art nach den Leistungen der gesetzlichen Krankenversicherung und der gesetzlichen Rentenversicherung entspricht, gilt nach $\S 7 a$ Abs. 6 SGB IV die sogenannte Amnestieregelung: Stellt die Rentenversicherung eine abhängige Beschäftigung als Ergebnis der Statusfeststellung fest, tritt die Versicherungspflicht nicht rückwirkend, sondern erst mit Bestandskraft des Feststellungsbescheids ein, d. h. erst zu dem Zeitpunkt, in welchem der Bescheid nicht mehr mit Rechtsmitteln angefochten werden kann.

Wird der Antrag aber erst bei einem bereits länger laufenden Beschäftigungsverhältnis gestellt, sollte sich der Praxisinhaber dringend vorab über die Höhe der Rückforderungen der Rentenversicherung klarwerden, die bei einem negativen Bescheid der Rentenversicherung blühen. Vorab muss also das Risiko im Einzelnen geklärt werden, welcher Betrag ggf. auf den Osteopathen zukommen kann. Auf keinen Fall sollte der Antrag auf Statusfeststellung unbedacht und ohne umfassende vorherige Beratung gestellt werden.

Zu beachten ist auch: Die Statusfeststellung ist nur für spätere Entscheidungen der Rentenversicherungen bindend, wenn sich die zu prüfenden Umstände nicht ändern und diese auch wahrheitsgemäß angegeben wurden. Sollte sich bei einer späteren Betriebsprüfung aber beispielsweise herausstellen, dass sich die Umstände der Beschäftigung verändert haben oder auch im Zeitpunkt der Statusfeststellung anders umgesetzt wurden, ist die Rentenversicherung an die vorherige Entscheidung nicht gebunden. 


\section{Lösungen}

\section{Wechsel zum Anstellungs- verhältnis}

Eine Lösung ist die Anstellung des freien Mitarbeiters. Ab diesem Zeitpunkt leisten der Arbeitgeber die Sozialversicherungsbeiträge (Arbeitgeberanteil) wie auch der Arbeitnehmer den Arbeitnehmeranteil, sodass eine Scheinselbstständigkeit ausgeschlossen ist. Ausgeschlossen ist hierdurch aber nicht das Risiko für die Vergangenheit. Im Gegenteil - bei einem Wechsel zum Anstellungsverhältnis ist die Scheinselbstständigkeit für die Vergangenheit sogar dokumentiert, wenn sich die Umstände der Beschäftigung nicht grundlegend geändert haben.

\section{Mietvertrag schließen}

Häufig wird empfohlen, anstelle eines sog. freien Mitarbeitervertrags einen Mietvertrag mit dem Beschäftigten zu schließen, um das Risiko der Scheinselbstständigkeit auszuschließen.

\section{Merke}

Der Abschluss eines Untermietvertrags mit dem in der Praxis Tätigen schließt das Risiko der Scheinselbstständigkeit allerdings nur dann aus, wenn der Untermieter in seiner Außendarstellung und der Umsetzung der eigenen Praxis völlig getrennt von der sonstigen Praxis des Vermieters agiert.

Es gelten also die gleichen Prüfungskriterien wie bereits aufgeführt.

\section{Gründung einer Gesellschaft}

Zur Vermeidung der Scheinselbstständigkeit kommt die Gründung einer sog. Berufsausübungsgemeinschaft (Gemeinschaftspraxis) infrage. Eine Gemeinschaftspraxis stellt regelmäßig eine Gesellschaft mit bürgerlichem Recht (GbR) dar. Gesellschaftszweck der GbR ist die gemeinsame Berufsausübung, im Fall der osteopathischen Praxis somit das Angebot osteopathischer Behandlungen. Die Umsetzung einer Gemeinschaftspraxis bedeutet, dass der bisherige freie Mitarbeiter Gesellschafter der GbR und damit Inhaber der Praxis wird.

Die Gemeinschaftspraxis ist strikt von der sog. Organisationsgemeinschaft oder Praxisgemeinschaft zu trennen. Letztere verfolgt nur den Gesellschaftszweck der gemeinsamen Nutzung von Räumlichkeiten, das Führen der osteopathischen Praxis erfolgt aber durch die Gesellschafter getrennt. Aus diesem Grunde ist diese Form der GbR auch keine Lösung, um der Scheinselbstständigkeit zu entgehen.
Vorsicht ist auch wegen des Risikos der sog. Scheingemeinschaftspraxis geboten.

Wird die Gesellschaft und somit die Gemeinschafspraxis nur gegründet, um ein Anstellungsverhältnis (Scheinselbstständigkeit) zu verdecken, drohen auch hier die beschriebenen Risiken. Mindestvoraussetzung für die Stellung eines Mitgesellschafters ist nach Auffassung des Bundessozialgerichts eine Beteiligung des Gesellschafters am wirtschaftlichen Risiko der Gesellschaft. Trägt ein Gesellschafter kein wirtschaftliches Risiko, etwa weil der betroffene Gesellschafter einen vom Erfolg der Praxis unabhängigen, festen Gewinnanteil erhält, liegt unter Umständen ein verdecktes Anstellungsverhältnis (Scheinselbstständigkeit) vor. Die Beteiligungsverhältnisse an der Gesellschaft können zwar unterschiedlich sein, ein Ausschluss eines Gesellschafters an dem Vermögen der Gesellschaft ist aber nicht zulässig. Keiner der Gesellschafter darf durch Direktionsrechte der Mehrheitsgesellschafter in der Dispositionsfreiheit eingeschränkt sein. Jeder Gesellschafter muss in beruflicher und persönlicher Hinsicht über eine ausreichende Handlungsfreiheit verfügen und in der Gesellschafterversammlung stimmberechtigt sein. Zudem achten die Gerichte da- 
rauf, dass auch der Minderheitengesellschafter gegenüber den sonstigen Bürokräften weisungsbefugt ist [14].

Eine Entscheidung des Landessozialgerichts Baden-Württemberg vom 23.11.2016 (Az. L 5 R 1176/15) macht das Risiko der sog. Scheingemeinschaftspraxis gut deutlich: Der Zahnarzt praktizierte gemeinsam mit einer Kollegin in einer Praxis. Die beiden Ärzte hatten hierfür eine Gemeinschaft bürgerlichen Rechts gegründet und einen „Gesellschaftsvertrag“ abgeschlossen. Dieser legte u.a. fest, dass die Ärztin 30\% ihrer Honorare erhielt. Den übrigen Überschuss aus den Einnahmen erhielt ihr Partner, nachdem er von diesen Einnahmen sämtliche Praxisausgaben beglichen hatte. Dazu gehörten u. a. die Miete, der Unterhalt der Praxis die Praxiseinrichtung gehörte allein dem Zahnarzt - und die Personalkosten. Die beiden Vertragspartner legten fest, dass sie gleichberechtigt und einander nicht weisungsbefugt seien. Im Rahmen einer Betriebsprüfung forderte der zuständige Sozialversicherungsträger den Arzt auf, für die Ärztin rückwirkend Sozialabgaben von mehr als $13000 €$ zu zahlen, da sie abhängig beschäftigt sei. Auch das angerufene Gericht sah ein abhängiges Beschäftigungsverhältnis: So trage die Zahnärztin kein wirtschaftliches Risiko und sei auch nicht am wirtschaftlichen Erfolg der Praxis beteiligt. Hinsichtlich der Sprechzeiten und der Urlaubsplanung müsse sie sich mit dem Zahnarzt und dem übrigen Praxispersonal absprechen. Erkranke sie länger als 6 Wochen, habe ihr Kollege die Befugnis, zulasten ihres Gewinnanteils einen Vertreter einzustellen. Umgekehrt gelte diese Regelung jedoch nicht.

\section{Kündigung der freien Mitarbeiter}

Die sicherlich drastischste Lösung zur Vermeidung einer Scheinselbstständigkeit in der Zukunft ist die Kündigung der freien Mitarbeiter zum nächstmöglichen Zeitpunkt. Ist die Betriebsprüfung noch nicht angesetzt, bedeutet dies bei der 4-jährigen Verjährungsfrist für Rückforderungsansprüche ein monatlich abnehmendes Risiko.

\section{Änderung im bestehenden Beschäftigungsverhältnis}

Besteht ein Beschäftigungsverhältnis schon über einen geraumen Zeitraum, sodass die Statusfeststellung im Rahmen der sog. Amnestieregelung nicht mehr möglich ist, stellt das Statusfeststellungsverfahren häufig ein erhebliches Risiko dar, das der Praxisinhaber verständlicherweise nicht eingehen will oder kann. Soll das Beschäftigungsverhältnis fortbestehen, ist es daher ratsam, das Risiko zu reduzieren, indem die vertragliche Regelung mit dem freien Mitarbeiter sowie auch die tatsächliche Umsetzung des Vertrags in der Praxis so umgeändert wird, dass bei einer Prüfung die Mehrzahl der oben genannten Kriterien für eine Selbstständigkeit sprechen.

\section{Fazit}

Zusammenfassend bleibt festzuhalten, dass die Beschäftigung freier Mitarbeiter in der osteopathischen Praxis ein ganz erhebliches Risiko für den Praxisinhaber, d. h. Auftraggeber des freien Mitarbeiters, darstellt. Jeder Praxisinhaber ist daher bei bestehender Beschäftigung freier Mitarbeiter sehr gut beraten, das Risiko im Einzelnen mit guter Beratung einzuschätzen, Lösungsmöglichkeiten in dem Einzelfall auszuarbeiten und ein Statusfeststellungsverfahren nur nach genauer Einschätzung des Risikos zu durchlaufen.

Besteht Bedarf, die bestehende Praxis um weitere Osteopathen zu erweitern, ist von dem Modell des freien Mitarbeiters aufgrund der bestehenden Risiken abzuraten. Insbesondere, wenn das Team ergänzt und die erweitere Praxis auch nach außen in Erscheinen treten soll, ist das Anstellungsverhältnis dringend vorzuziehen.

\section{Literatur}

[1] BSG, Urteil vom 18.11.2015, Az. B 12 KR 16/ $13 \mathrm{R}$

[2] BSG, Urteil vom 24.03.2016, Az. B 12 KR 20/ $14 \mathrm{R}$

[3] BSG, Urteil vom 29.08.2012, Az. B 12 KR 25/ 10

[4] Landessozialgericht für das Land NordrheinWestfalen, Urteil vom 26.04. 2016, Az. L 8 R $744 / 15$ B ER
[5] BSG, Urteil vom 24.03.2016, Az. B 12 KR 20/ $14 \mathrm{R}$

[6] BSG, Urteil vom 31. Mai 1978 - 12 RK 25/77

[7] SozR $2200 \S 1229 \mathrm{Nr} 8$, Rndnr. 22

[8] Landessozialgericht Niedersachsen-Bremen, Urteil vom 24. 09. 2014, Az. L 1 KR 351/12

[9] BSG, Urteil vom 28.09.2011, Az. B 12 R 17/ 09 R; BSG, Urteil vom 18.11.2015, Az. B 12 KR $16 / 13 R$

[10] BSG, Urteil vom 24.03.2016, Az. B 12 KR 20/ $14 \mathrm{R}$

[11] Landessozialgericht Niedersachsen-Bremen, Urteil vom 24.09.2014, Az. L 1 KR 351/12; Bayrisches Landessozialgericht, Beschluss vom 13.02.2014, Az. L 5 R 1180/13 B

[12] BSG, Urteil vom 24.03.2016, Az. B 12 KR 20/ $14 \mathrm{R}$

[13] Bayerisches Landessozialgericht, Urteil vom 13. 02. 2014, Az. L 5 R 1180/13 B ER

[14] Landessozialgericht Niedersachsen-Bremen, Urteil vom 17.05.2017, Az. L 2 R427/15

[15] BSG, Urteil vom 23.06.2010 - B 6 KA 7/09 R

[16] Ratzel R, Luxemburger B. Handbuch Medizinrecht. 13. Aufl. München: Beck; 2015

[17] Jaeger L. Patientenrechtegesetz. Kommentar. Karlsruhe: Verlag Versicherungswirtschaft; 2013

[18] Palandt O. Bürgerliches Gesetzbuch. 75. Aufl. München: Beck; 2016

Über die Autoren

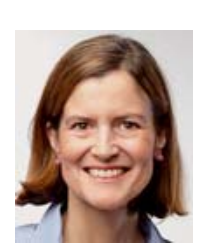

Dr. iur. Sylke Wagner-Burkard

ist als Rechtsanwältin in eigener Kanzlei in der Rhön mit Schwerpunkt Recht der Heilberufe tätig. Als Justitiarin des Verbandes der Osteopathen Deutschland e.V. vertritt sie die rechtlichen Interessen des Verbandes und berät die mehr als 4000 Mitglieder.

Korrespondenzadresse

Dr. iur. Sylke Wagner-Burkard, LL.M.

Rechtsanwältin

Neuwart 48

36163 Poppenhausen (Wasserkuppe)

info@kanzlei-wagnerburkard.de

Bibliografie

DOI https://doi.org/10.1055/s-0043-114764

DO - Deutsche Zeitschrift für Osteopathie 2017; 15: 35-40 ๑ Georg Thieme Verlag KG Stuttgart . New York I ISSN 1610-5044 